\title{
Evaluation of Late Gadolinium Enhancement and Cardiac Cine-MRI Based on Artificial Intelligence in a Patient with Selenium Deficiency Cardiomyopathy (Keshan Disease): A Case Report
}

\author{
Wei-Feng Yuan (iD ${ }^{1,2}$ and Xin-Xiang Zhao (iD) ${ }^{1, *}$ \\ ${ }^{1}$ Department of Radiology, The Second Affiliated Hospital, Kunming Medical University, Kunming, China \\ ${ }^{2}$ Department of Radiology, The First Affiliated Hospital, Chengdu Medical College, Chengdu, China \\ "Corresponding author: Department of Radiology, The Second Affiliated Hospital, Kunming Medical University, The 374th Dianmian Road, Wuhua District, Kunming, China, \\ +86-1388829784, Email: zhaoxinxiang0815@hotmail.com
}

Received 2019 April 02; Revised 2020 January 29; Accepted 2020 February 09.

\begin{abstract}
Keshan disease is an endemic cardiomyopathy with unknown causes. The disease mainly invades the myocardium, manifested by myocardial degeneration, necrosis, repair and other changes. MRI can objectively reflect the above pathological mechanism and characteristics, but there is no relevant literature report at present. A 51-year-old male patient was hospitalized and suspected of suffering from rare cardiomyopathy. Cardiovascular magnetic resonance imaging (CMRI) showed late gadolinium enhancement (LGE) in the areas other than the anterior wall of the left ventricle, accompanied by irregular epicardial LGE mainly in the left ventricular apex. Results of left ventricular function parameters calculated by artificial intelligence (AI) were as follows: end-systolic volume (ESV) $98.14 \mathrm{~mL}$, end-diastolic volume (EDV) $121.59 \mathrm{~mL}$, ejection fraction (EF) 35.13\%, end-diastolic dimension (EDD) $47.49 \mathrm{~mm}$, fraction shortening (FS) 24.83\%, myocardial mass (MM) $62.74 \mathrm{~g}$, cardiac output (CO) $3.49 \mathrm{~L} / \mathrm{min}$, stroke volume (SV) $42.71 \mathrm{~mL}$, and cardiac index (CI) $3.09 \mathrm{~L} / \mathrm{min} \cdot \mathrm{m}^{2}$. According to the results of CMRI-LGE and left-ventricular functional parameters based on AI, Keshan disease manifested as enlarged morphology, abnormal cardiac function parameters, decreased myocardial cell activity, degeneration and necrosis, and myocardial interstitial fibrosis formation, which might help to diagnose the disease after excluding ischemic heart disease and primary cardiomyopathy.
\end{abstract}

Keywords: MRI, Artificial Intelligence, Cardiomyopathy

\section{Introduction}

Keshan disease is an endemic cardiomyopathy with unknown causes (1). It can be divided into four clinical types: acute, sub-acute, slow and potential. The disease mainly invades the myocardium, manifested by myocardial degeneration, necrosis, repair and other changes. MRI can objectively reflect the above pathological mechanism and characteristics, but there is no relevant literature report at present. Acute Keshan disease mainly occurs in northern China. It occurs in women during childbearing period. Subacute Keshan disease mainly occurs in southwestern China and in children aged 3 - 8 years (2). Keshan disease is closely related to dietary nutrition. The single dietary structure, low nutrition and low selenium increase the incidence of Keshan disease. The clinical manifestations of Keshan disease usually include general discomfort, heart distress, chest tightness, nausea, paleness, lower limb edema and other symptoms and signs.

\section{Case Presentation}

A 51-year-old male patient was sent to our hospital for about 3 weeks because of chest tightness, fatigue and cough. He was once ill with hepatitis B virus and underwent a liver biopsy, indicating mild to moderate active hepatitis and fibrosis without obvious jaundice. One year ago, he had chest pain for unknown inducement and was accompanied by ventricular arrhythmia, and was treated with medication to prevent arrhythmia and ventricular remodeling. Thyroid function tests were normal. There was no clinical or pathological evidence of hypertension. Two days after admission, he gradually fell into a coma, by heart failure associated with ventricular arrhythmia. Resuscitative measures were initially successful. Troponin value was significantly increased $(0.74 \mu \mathrm{g} / \mathrm{L})$, with a slight increase in B-type natriuretic peptide $(143 \mathrm{pg} / \mathrm{mL}$ ) in laboratory blood tests. His resting electrocardiograph showed raised ST segments in all leads. The provisional clinical diagnosis 
was probable stress-induced cardiomyopathy leading to spastic ischemia of the coronary artery, while coronary atherosclerotic heart disease was not the first consideration. The patient gave informed consent to the work, and all examinations were conducted in accordance with the guiding principles of Medical Ethics Committee of the First Affiliated Hospital of Chengdu Medical College.

No sign of heart disease was found in the previous chest radiograph, 24-hour electrocardiography (ECG) monitoring or emergency bedside echocardiography. To further assess the origin of troponin-elevation, a cardiovascular magnetic resonance imaging (CMRI) study was performed (3.0 T, Ingenia, Philips Medical Systems, Netherlands). Late gadolinium enhancement (LGE) was considered to be a reference standard for evaluating myocardial survival and myocardial interstitial fibrosis. It was performed 10 minutes after gadolinium-based MRI contrast agent injected, which might provide important information for the prognosis of non ischemic cardiomyopathy and myocarditis (3). In order to prove that there was a feasible region of high signal intensity caused by edema, the steady-state free precession sequence of cine MRI was used for the long axis of four and two cavities, the fast spin echo sequence of black blood was used for the short axis plane, the double inversion recovery was used to suppress the blood signal, and the additional inversion recovery pulse was used to suppress the fat signal. Then, a series of short axis slices were obtained by cine MRI sequence to evaluate regional and global biventricular functional dynamics. After perfusion imaging, gadolinium diethylenetriamine pentaacetic acid (DTPA) of $0.1 \mathrm{mmol} / \mathrm{kg}$ was administered at a rate of $2.0 \mathrm{~mL} / \mathrm{s}$. After 10 minutes, inverse recovery (IR) and segmented gradient echo (LGE-MRI) sequences were used to obtain lge-mri images with each layer holding breath. These typical settings were as follows: in the short axis view, a group of eight adjacent slices had the same orientation as perfusion imaging. In the case of no cross gap, the slice thickness was $10 \mathrm{~mm}$, the field of view (FOV) was $320 \mathrm{~mm}$, the repetition time (TR)/echo time (TE) was 6.1/3.0 MS, the turning angle was $25^{\circ}$, and the matrix was $192 \times$ 160. CMRI showed LGE in the areas other than the anterior wall of the left ventricle, accompanied by irregular epicardial LGE mainly in the left ventricular apex. (Figure 1AC). This rather typical enhancement pattern excluded coronary atherosclerotic heart disease. In addition, it was neither like restrictive cardiomyopathy, nor like the decompensated period of hypertensive heart disease. No sign of cytotoxic edema or interstitial edema was found in the T2weighted sequences with fat suppression in four-chamber and two-chamber long-axis. Viral myocarditis did not accord with the above manifestations (4). Some rare cardiomyopathies such as alcoholic heart disease could not be completely ruled out for the time being. A series of short axis sections were obtained by cine MRI sequence to evaluate the local and global biventricular functional dynamics. According to the American Heart Association (AHA), 16 segments of left ventricular myocardium (excluding the 17th segment) were quantitatively analyzed for global left ventricular function parameters and wall thickening rates (5). Drawing of the contour of endocardium and epicardium on the short axis plane of cine MRI image was based on artificial intelligence (Figure $2 \mathrm{~A}-\mathrm{C}$ ). Cardio DL, the first CMRI artificial intelligence software approved by FDA and certified by CE in Europe, was applied to post-processing analysis of CMRI images in this study. Results of left ventricular function parameters calculated by artificial intelligence (AI): end-systolic volume (ESV) $98.14 \mathrm{~mL}$, end-diastolic volume (EDV) $121.59 \mathrm{~mL}$, ejection fraction (EF) 35.13\%, enddiastolic dimension (EDD) $47.49 \mathrm{~mm}$, fraction shortening (FS) 24.83\%, myocardial mass (MM) 62.74 g, output (CO) $3.49 \mathrm{~L} / \mathrm{min}$, stroke volume (SV) $42.71 \mathrm{~mL}$, cardiac and cardiac index (CI) $3.09 \mathrm{~L} / \mathrm{min} . \mathrm{m}^{2}$. It was found that the $a b-$ normality of cardiac structure and function mainly manifest as myocardium thinning, chamber volume enlarging, systolic dysfunction and diastolic dysfunction. Therefore, rare cardiomyopathy was suspected. Through a series of systematic clinical history and more examinations, it was found that the serum selenium value was abnormally decreased (0.4, the reference value ranged from 0.9 to 2.0 $\mu \mathrm{mol} / \mathrm{L})$. Histologic examination of the myocardium of the left ventricle by biopsy showed myocardial cells with abnormal manifestations of different sizes and shapes. There were many fibrous tissues in the myocardial interstitium, which proved the degeneration and necrosis of myocardial cells. Viral infection was fully confirmed by immunohistochemical analysis of T-lymphocytes and monocytes. Monoclonal antibody 5-D8/1 was analyzed by immunohistochemistry, and in situ hybridization with enterovirusspecific probes (digoxin labelled) was performed (Figure 3). The presence of VP1 and/or viral genomic RNA was detected in the samples collected and screened. The diagnosis of Keshan disease was based on selenium deficiency and the above-mentioned myocardial microscopic manifestations.

\section{Discussion}

The characteristic signs of enterovirus were identified in degenerated myocardial cells and interstitial fibrous tissues, and confirmed its important role in the pathological mechanism of Keshan disease, and also expressed positive signs in reverse transcription nested polymerase chain reaction. Keshan disease was highly similar to dilated cardiomyopathy, such as morphological changes, abnormal 

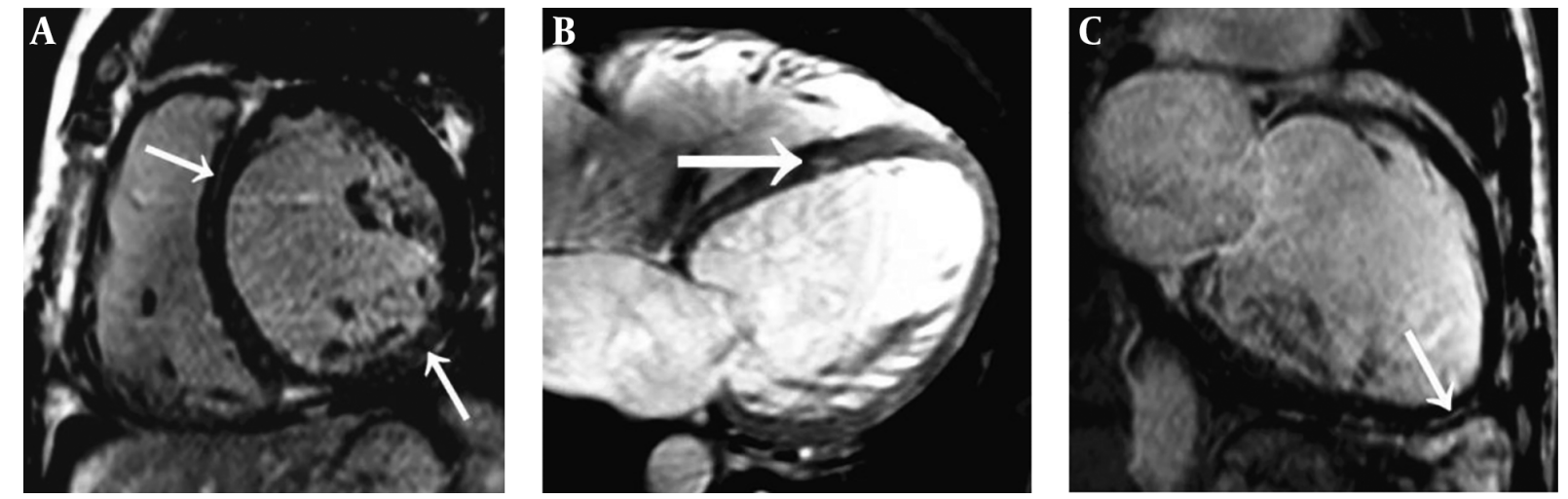

Figure 1. A 51 year-old man with a diffuse form of Keshan disease. A, Short-axis; B, Four-chamber; and C, Long-axis delayed-enhancement images. Nodular midmyocardial late gadolinium enhancement (LGE) (arrows in A and B) in the areas other than the anterior wall of the left ventricle, accompanied by irregular epicardial LGE (arrow in C) mainly in the left ventricular apex.
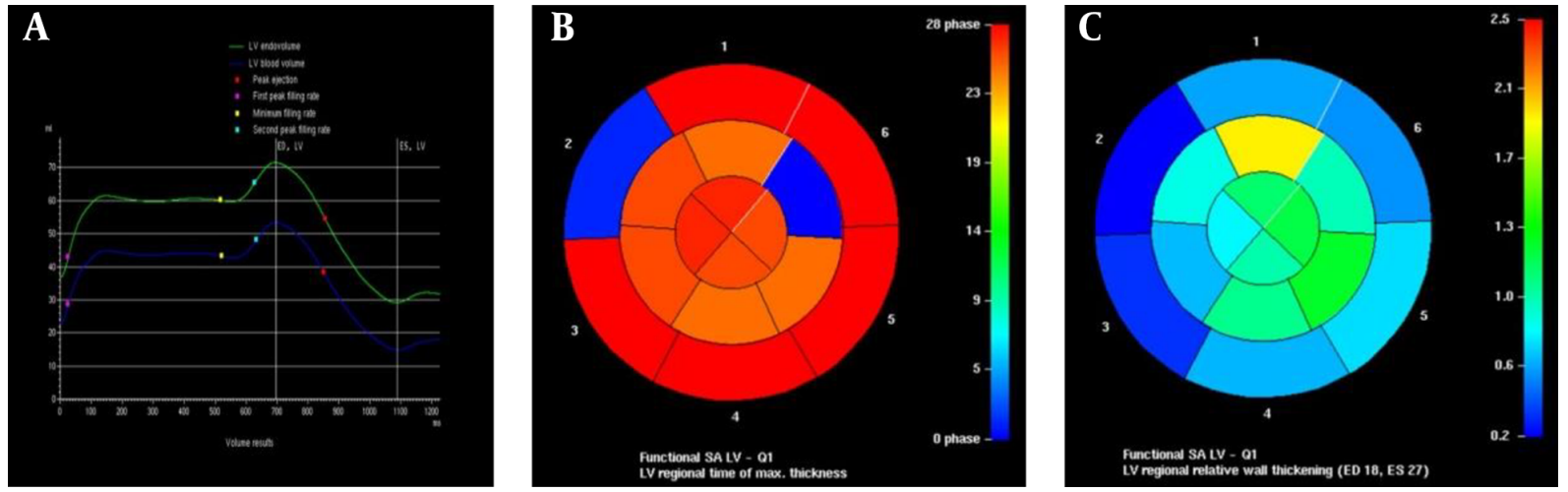

Figure 2. A, Time-volume quantitative curve in the left ventricle; B, Color quantization mapping regional time of thickness ${ }_{\max }$ in the left ventricle; and C, Color quantization mapping regional relative wall thickness in the left ventricle. Ventricular arrhythmia is demonstrated in A. High quantitative value indicates warm color, while low quantitative value indicates cold color in both B and C.

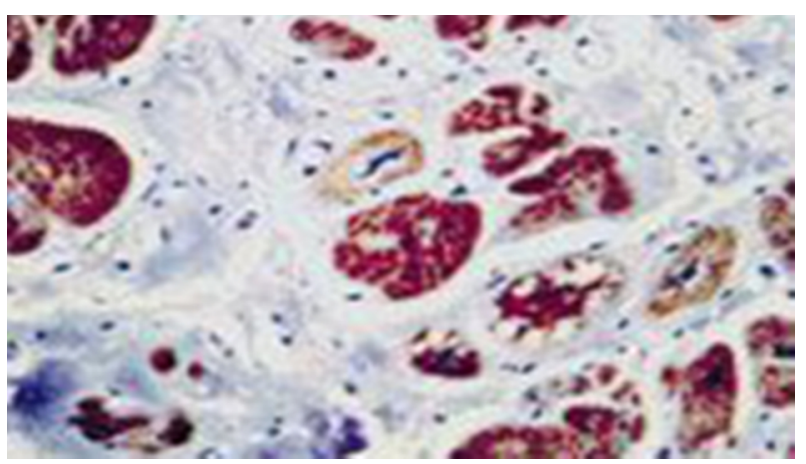

Figure 3. Monoclonal antibody 5-D8/1 was analyzed by immunohistochemistry, and in situ hybridization with enterovirus-specific probes (digoxin labelled) was performed. changes of cardiac function parameters, decreased myocardial cell activity, degeneration and necrosis, and myocardial interstitial fibrosis formation, which were easy to be misdiagnosed. Within four weeks, the patient's condition was unstable and gradually deteriorated. However, after taking selenium supplements and conventional arrhythmic drugs in line with medical treatment dose, the patient gradually recovered and was then discharged from hospital. As far as we know, this is the first reported case in which multisequencing CMRI data was obtained in the same patient with Keshan disease, which is an endemic cardiomyopathy that has occurred in China and to some extent still occurs today (6). It is suggested that patients with suspected Keshan disease should undergo CMRI multisequence examination (especially in morphology, left ventricular function parameter analysis and myocardial activity evaluation), because it may have great potential diag- 
nostic and prognostic evaluation value.

\section{Footnotes}

Authors' Contributions: Study concept and design: XinXiang Zhao. Analysis and interpretation of data: Wei-Feng Yuan. Drafting of the manuscript: Wei-Feng Yuan. Critical revision of the manuscript for important intellectual content: Xin-Xiang Zhao. Statistical analysis: Wei-Feng Yuan

Conflict of Interests: All the authors declare no conflict of interests in this study.

Ethical Approval: This study has passed the examination of the medical Ethics Committee of the hospital.

Funding/Support: This study was supported in part by grant 2017FE468-178 from the Applied Basic Research on projects in Yunnan Province and grant D-201646 from the Yunnan Province Medical Subject Leaders Training project and grant 2015HB068 from the young and middle-aged Technical Academic Leaders Training project in Yunnan province and grant CYZ17-34 from Natural Science Foundation of Chengdu Medical College.

Informed Consent: The patient has signed informed consent in this study.

\section{References}

1. Zhou B, He S, Wang XI, Zhen X, Su X, Tan W. Metabolism of arachidonic acid by the cytochrome P450 enzyme in patients with chronic Keshan disease and dilated cardiomyopathy. Biomed Rep. 2016;4(2):2515. doi: 10.3892/br.2015.563. [PubMed: 26893848]. [PubMed Central: PMC4734242].

2. Liu H, Yu F, Shao W, Ding D, Yu Z, Chen F, et al. Associations between selenium content in hair and Kashin-Beck disease/Keshan Disease in children in Northwestern China: A prospective cohort study. Biol Trace Elem Res. 2018;184(1):16-23. doi: 10.1007/s12011-017-1169-x. [PubMed: 28983831].

3. Cummings KW, Bhalla S, Javidan-Nejad C, Bierhals AJ, Gutierrez FR, Woodard PK. A pattern-based approach to assessment of delayed enhancement in nonischemic cardiomyopathy at MR imaging. Radiographics. 2009;29(1):89-103. doi: 10.1148/rg.291085052. [PubMed: 19168838].

4. Mahrholdt H, Wagner A, Deluigi CC, Kispert E, Hager S, Meinhardt G, et al. Presentation, patterns of myocardial damage, and clinical course of viral myocarditis. Circulation. 2006;114(15):1581-90. doi: 10.1161/CIRCULATIONAHA.105.606509. [PubMed: 17015795].

5. Cerqueira MD, Weissman NJ, Dilsizian V, Jacobs AK, Kaul S, Laskey WK, et al. Standardized myocardial segmentation and nomenclature for tomographic imaging of the heart: A statement for healthcare professionals from the Cardiac Imaging Committee of the Council on Clinical Cardiology of the American Heart Association. JAm Soc Echocardiography. 2002;15(5):463-7. doi:10.1067/mje.2002.123374.

6. Hou J, Wang T, Liu M, Li S, Chen J, Liu C, et al. Suboptimal selenium supply-a continuing problem in Keshan disease areas in Heilongjiang province. Biol Trace Elem Res. 2011;143(3):1255-63. doi: 10.1007/s12011-011-8961-9. [PubMed: 21253891]. 